\title{
MEASUREMENT SYSTEM FOR LOW FORCE AND SMALL DISPLACEMENT CONTACTS
}

\author{
Beth L. Pruitt, Woo-Tae Park, Thomas W. Kenny
}

Dept. of Mechanical Engineering, Stanford University,

Stanford, CA 94305, pruitt@,stanford.edu

\begin{abstract}
In order to further miniaturize electrical contacts for novel IC packaging methods and MEMS scale relays, combined measurements of the electrical and mechanical properties of thin film metallization used in low-force contacts is required. Comprehensive data to design these low-force electrical contacts is lacking because no system is readily available for measuring forces, displacements, and electrical resistance at the levels of interest. A new measurement system is designed to characterize the low force behavior of thin gold films by flexibly varying these conditions and implementing an interferometer. This new MEMS/Materials characterization system is capable of measuring displacements and deformations down to $0.1 \AA$, contact forces from $\mathrm{mN}$ to $\mathrm{nN}$, and contact resistance from $\mathrm{m} \Omega$ to $\mathrm{k} \Omega$, and represents the first complete system capable of simultaneous measurements of all these parameters.
\end{abstract}

\section{INTRODUCTION}

The rapid development of integrated circuits requires denser packaging for higher integration. As the line width and pitch for interconnects and packaging pads shrink, the size of electrical contacts for packaging must also decrease to accommodate smaller packages and minimize the number of redistribution layers and the area required to transition to external or off-chip connections. For these reasons, the electronics packaging and testing industry is developing new methods to decrease the size of electrical contacts. One approach is the MicroSpring ${ }^{\mathrm{TM}}$ interconnect developed by Formfactor, Inc. and shown in Figure 1. Shrinking the size and force applied by these interconnects while maintaining good electrical contact is desirable. MicroSprings ${ }^{\mathrm{TM}}$ are used in probe cards for testing or can be integrated directly on the wafer to act as both the first and second level interconnect for packaging. To make these pressure-mated low force interconnects reliable, complete data for electrical and mechanical properties of contacts made with thin-film metallization are required.

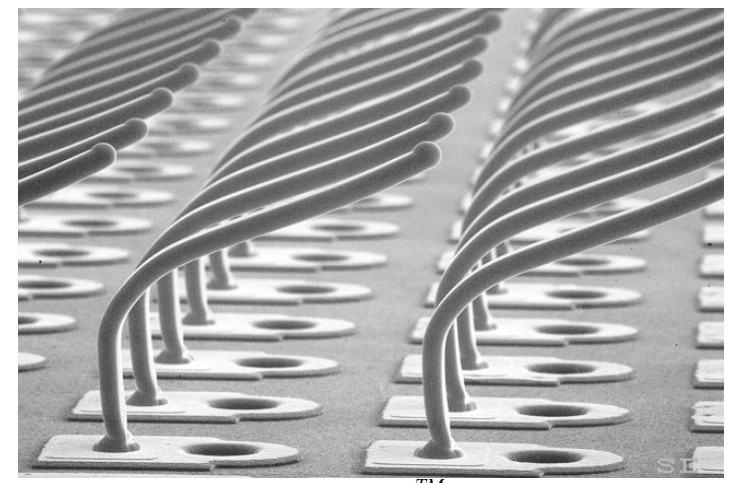

Figure 1. FormFactor MicroSprings ${ }^{T M}$.
Low force and small area contacts have been studied previously for very specific applications and materials systems. From the previous work, thin film properties have been found to vary substantially from bulk properties. Discrete results for a single device configuration, load, and material choice are reported for MEMS relays [1-4] or limited sets of thin films[5-7]. The majority of low force contact data available utilized force balance systems without continuous and synchronous data collection and is from bulk materials[8-12].

This paper presents a unique measurement system, as shown schematically in Figure 2, for evaluating thin film contacts and allows easy variation of loading, displacement, and current during the test. Forces are measured on a piezoresistive cantilever [13], which is stepped into contact with a sphere metallized identically to the contact pad on the cantilever. Stepwise displacement is controlled with a piezoelectric actuator with a theoretical resolution of $1 \mathrm{~nm}$ and range of $100 \mu \mathrm{m}$. The contact displacement is directly measured with an interferometer shown in Figure 3 and discussed later[14]. The materials tested in the initial experiments were thin films of evaporated gold with varied thickness[15]. Additonal tests with variation of thin film properties through manufacture method have since been conducted with improved accuracy in the revised experimental setup.

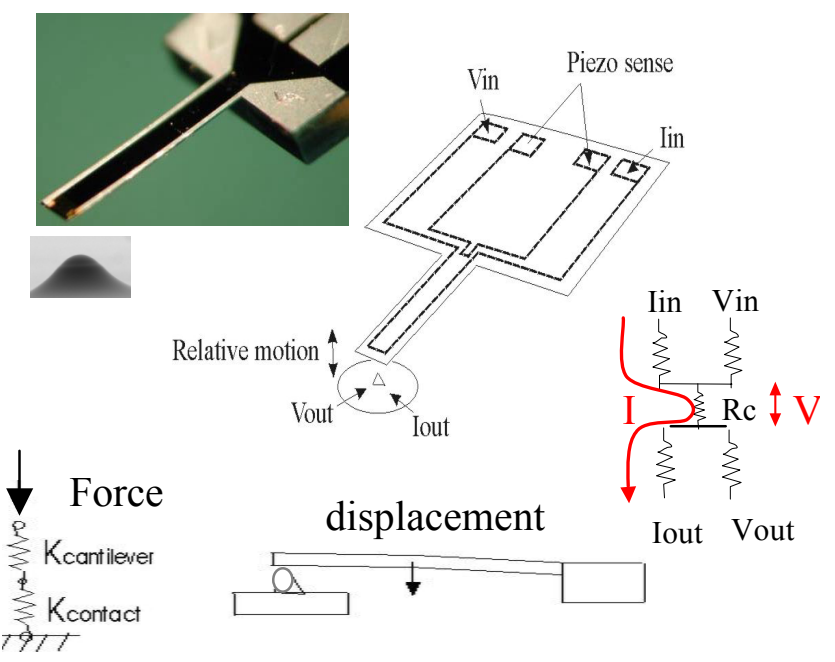

Figure 2. Piezoresistive cantilever and metallized contact sphere top pictured left. Measurement schematic depicts relationship to mechanical and electrical models used to extract force, displacement, and contact resistance $(R c=V / I)$ from the measurement.

Travel support has been generously provided by the Transducers Research Foundation and by the DARPA MEMS and DARPA BioFlips programs. 


\section{EXPERIMENT}

\section{Design and Characterization of Cantilevers}

Piezoresistive cantilever sensors were fabricated in order to measure force and contact resistance simultaneously. Cantilever geometry (width, length and thickness) and doping level/pattern were optimized to measure the desired force range $(\mathrm{mN} \sim \mathrm{nN})$. The sensor and the spherical contact "tip" are metallized with plated, sputtered and evaporated gold of varied thickness. Two aluminum traces on the cantilever along with the two electrical leads at the tip enable 4-wire contact resistance measurement when the sensor makes contact with the tip. The resonant frequency, effective spring constant, and calibration factor for voltage to force transformation is derived by mechanically resonating the cantilever and measuring tip displacement and piezoresistor voltage. The transformations are made using linear beam theory. Details of design and fabrication are discussed in a previous paper[13]. The contact geometry investigated is a sphere on flat because it is easily modeled, controlled, and characterized. Glass and polystyrene spheres of 50 and $100 \mu \mathrm{m}$ diameter were potted in photoresist spun on a 4-inch wafer for use as contact tips of different compliance. This process creates a spherical tip protruding from a concave sloped shoulder that enables uniform metallization, also shown in Figure 2. The tips are subsequently metallized with varying thickness of gold using different manufacturing methods.

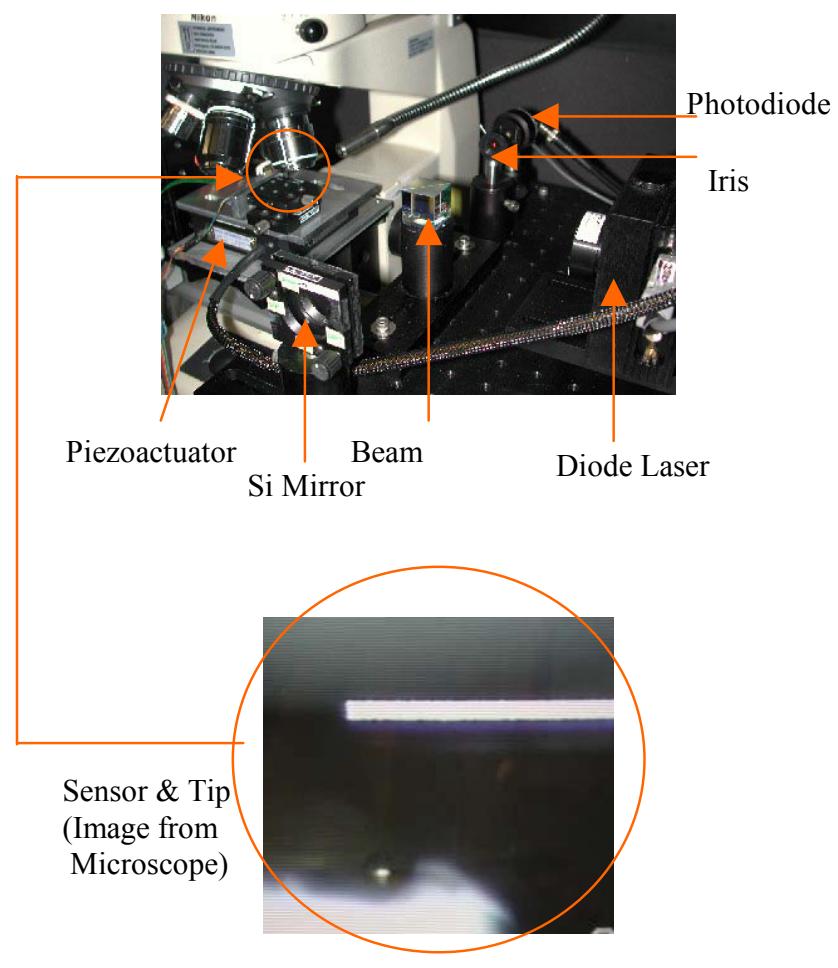

Figure 3. Measurement system incorporates a customized piezoresistive cantilever to measure force, PolytecP-731 2-axis nanopositioner and E515 closed loop controller with capacitive feedback to apply displacements, and a free space Michelson interferometer for measuring contact displacement.

The noise spectrum of the piezoresistive force sensor is shown in Figure 4; the noise floor of $80 \mathrm{pN}$ at $1 \mathrm{~Hz}$ is not limiting. Resolution is limited more by drift over the course of a full loading and unloading cycle. However, good resolution during contact initiation allows characterization of small force mechanical properties.

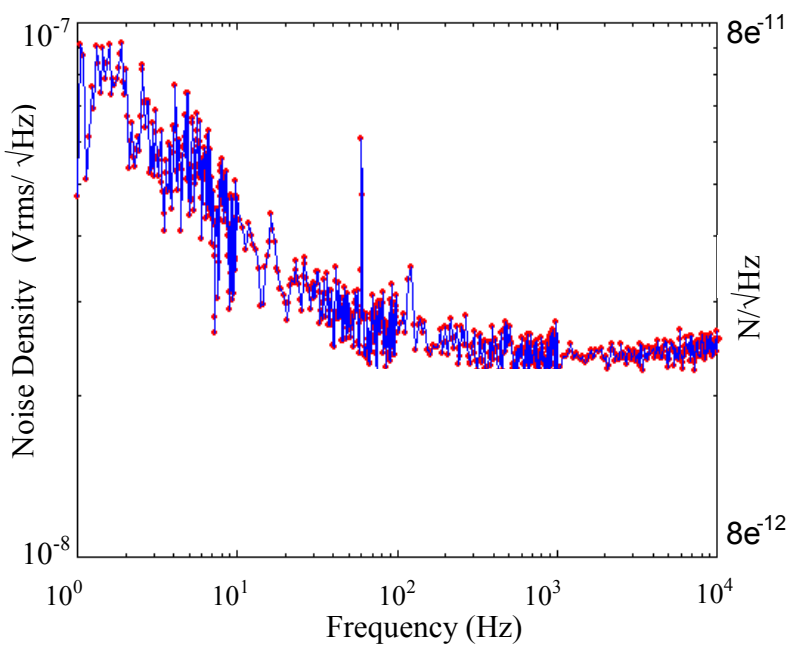

Figure 4. Noise data for piezoresistor is collected on an HP89410A vector signal analyzer. The piezoresistor noise over the frequency range $10^{-1}$ to $10^{5}$ is dominated by $1 /$ f noise below $1 \mathrm{kHz}$. Noise at $1 \mathrm{~Hz}$ is less than $0.1 \mathrm{uV} / \sqrt{ } \mathrm{Hz}$ or $80 \mathrm{pN} / \sqrt{\mathrm{Hz}}$.

\section{Contact Resistance Measurements}

Experiments conducted with the first experimental setup provided some baseline guidance for improvement and data on the effect of cleaning procedures[15]. In the revised setup, contact resistance was investigated with varying manufacture methods. Gold films were manufactured by evaporation, sputtering, hard gold plating, and soft gold plating

Figure 5 shows a set of data from films of different manufacture methods. The contact resistance is measured as a function of applied force during the applied loading. For the parameters of deposition used, evaporated gold provides a lower contact resistance than the other films. This difference in behavior is correlated with microstructure and surface roughness. From the figure, we can quantify the adhesion force of each film. It is $20 \mu \mathrm{N}$ for evaporated gold, $60 \mu \mathrm{N}$ for hard plated gold, and $600 \mu \mathrm{N}$ for sputtered gold.

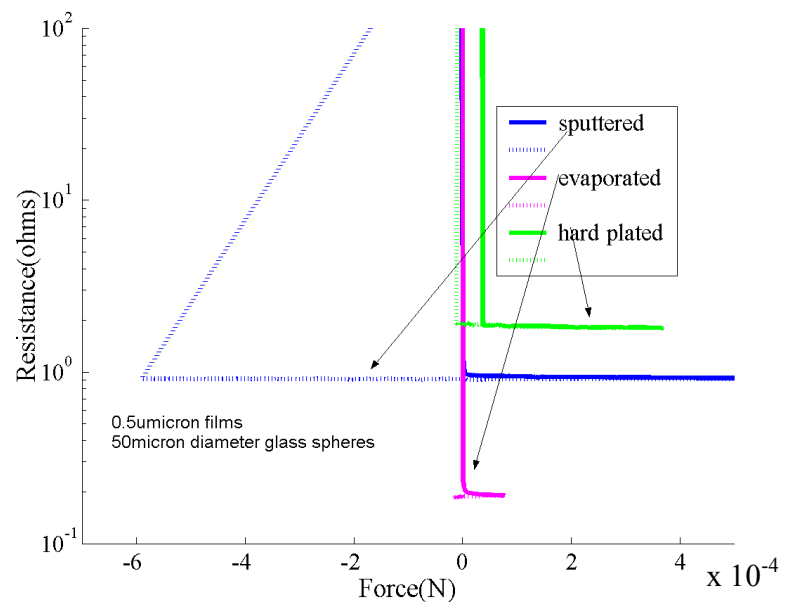

Figure 5. Contact tests on 50um diameter glass contact spheres, $0.5 \mu \mathrm{m}$ gold film deposited by sputtering, evaporating, or hard plated gold. The solid line represents the loading data and the dashed line is unloading. 


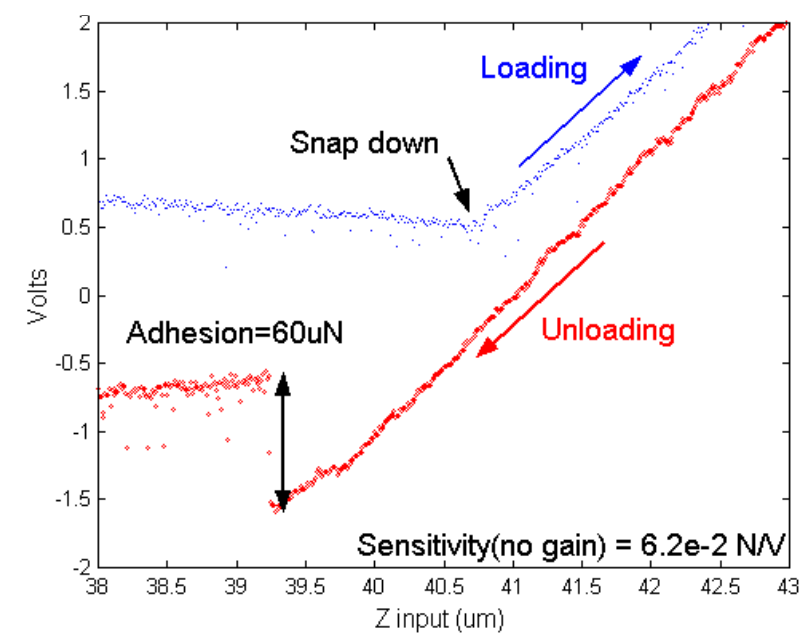

Figure 6. PiezovoltageX1000 vs. input position for $1800 \AA$ evaporated gold film. Force is extracted from the piezoresistive cantilever calibration and features like pull off adhesion and snap down can be extracted. These are correlated with applied current, film thickness, material properties, and substrate type.

In Figure 6, the snap down force and adhesion force are clearly visible by zooming in data from piezovoltage vs. input displacement.

\section{Contact Displacement Measurement}

"Contact displacement" as shown in Figure 7 is the amount of the total deformation of the tip and the cantilever when force is applied to the contact. The elastic property of thin films can be characterized by measuring the contact displacement along with the corresponding force data. Nanoindentation is the appropriate method for extracting material properties, however it does not have the capabilities to measure electrical properties concurrently. In order to directly measure contact displacement, a Michelson interferometer is incorporated to the existing measurement system[14]. The configuration of the interferometer is shown in Figure 8. The back of the cantilever sensor replaces one the mirrors of a typical Michelson interferometer to measure the movement of the cantilever.

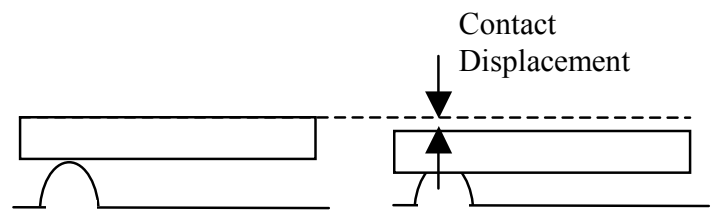

Figure 7. Definition of a "contact displacement". In the figure, the contact area is exaggerated, however the gold films on both sides are deformed during contact.

The movement of the cantilever produces a sinusoidal wave of intensity at the detector until the cantilever makes contact with the tip. Because the signal is a sinusoidal wave, the sensitivity is not a constant. The sensitivity of the interferometer as a contact displacement sensor depends on the position of the instant of contact relative to the sinusoidal sensitivity curve. If the contact is at the maxima or minima of the sine wave, the sensitivity will be zero. The maximum sensitivity will be halfway between maxima and minima. In order to get maximum sensitivity, the contact must occur in that region. However, it is impossible to predict the contact point at the start of the test, and not every contact test will yield usable interferometer data.

Figure 9 is the noise spectrum of the interferometer with a stationary cantilever. There are some high peaks between 10 and $100 \mathrm{~Hz}$, which is due to some random mechanical noise of the system. In spite of some random noise of the system we can see that the noise at $1 \mathrm{~Hz}$ is less than $1 \mathrm{mV} / \sqrt{\mathrm{Hz}}$, therefore combining with the sensitivity obtained from Figure 10, the theoretical noise floor is less than $10^{-1} \mathrm{~nm} / \sqrt{\mathrm{Hz}}$ at $1 \mathrm{~Hz}$ for a typical measurement.

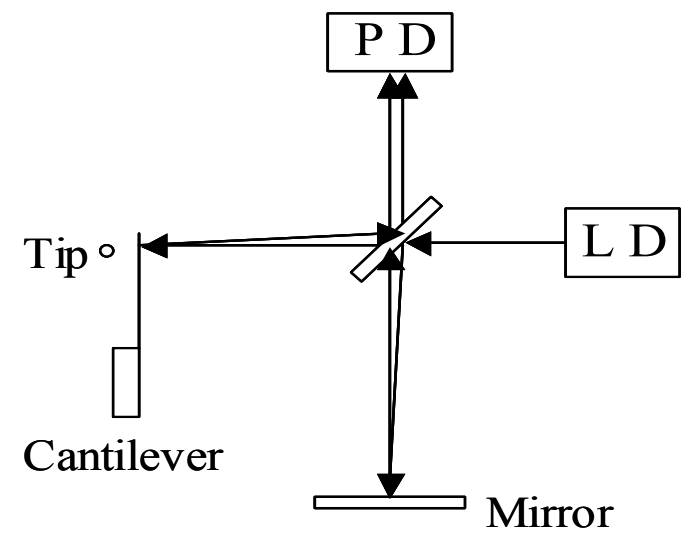

Figure 8. Schematic of free space Michelson interferometer and displacement measurement back. A Hitachi HL6312G 635nm laser diode (LD) with Melles-Griot 06DLD163 controller and Thorlabs PDA500 amplified GaAsP photo diode (PD) are used with a prism beam splitter, polished Si mirror, to measure fine displacement of the surface of the Si cantilever over the first several microns of contact.

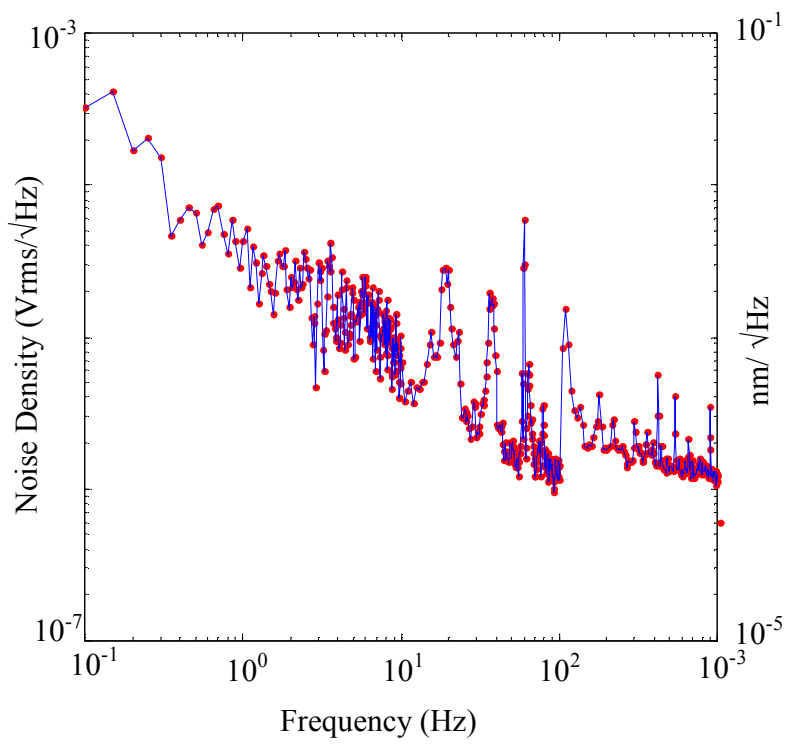

Figure 9. Noise data for interferometer are collected on an HP89410A vector signal analyzer.

Figure 10 shows a set of data from a contact of a silicon cantilever $(\mathrm{K}=72.1 \mathrm{~N} / \mathrm{m})$ on a polystyrene sphere with evaporated gold films. Although the movement of the piezoelectric actuator is not ideal and produces vibration, we can get an accurate measurement by averaging data. 


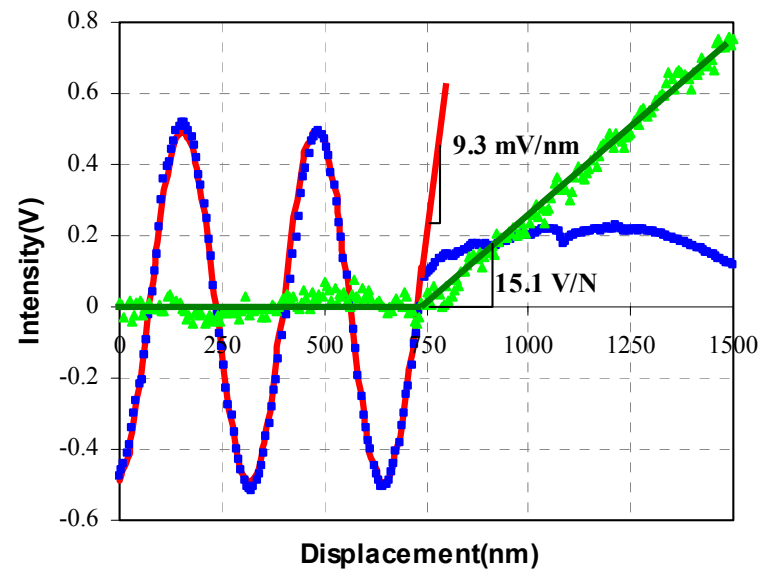

Figure 10. Data from contact of a silicon cantilever on a polystyrene sphere with $0.5 \mu \mathrm{m}$ evaporated gold films. The sinusoidal trace is the interferometer data with a curve fit, the linear flat to ramp trace is the piezovoltage with gain 1000.

From the data points shown in square dots in the figure, a sine curve is carefully fitted. The next step is to find the actual contact point by extrapolating the curve before contact and the curve after the contact. Differentiating the fitted curve at the contact point produces the sensitivity of the interferometer. In the figure, a sensitivity of $9.25 \mathrm{mV} / \mathrm{nm}$ was obtained by this method. The last step is to divide the voltage change by the sensitivity to get the displacement of the corresponding force. From this set of data, it can be shown that at a force of $5 \mu \mathrm{N}$, the measured contact displacement is $4.8 \mathrm{~nm}$. The contact displacement of the polystyrene sphere on a silicon flat surface is predicted from Hertzian elastic theory for the mutual approach of distant points in the solid as

$$
\delta=\frac{a^{2}}{r}=\left(\frac{9 P^{2}}{16 r E^{* 2}}\right)^{1 / 3}
$$

where,

$$
\begin{aligned}
& E^{*}=\frac{1-v_{1}^{2}}{E_{1}}+\frac{1-v_{2}^{2}}{E_{2}} \\
& \delta=\text { mutual approach of distant points on two } \\
& \text { sides of the contact }(\mathrm{m}) \\
& \mathrm{a}=\operatorname{radius} \text { of Hertzian elastic contact area }(\mathrm{m}) \\
& \mathrm{r}=\operatorname{radius} \text { of the sphere }(\mathrm{m}) \\
& \mathrm{P}=\operatorname{load}(\mathrm{N}) .
\end{aligned}
$$

Using the properties given in Table 1 and (1), the predicted displacement is $4.3 \mathrm{~nm}$. The difference in displacements is attributed to the deformation of the gold films.

\begin{tabular}{|l|c|c|}
\hline & Modulus, $\mathrm{E}(\mathrm{Pa})$ & Poisson's ratio, $v$ \\
\hline silicon & $2.00 \mathrm{E}+11$ & 0.23 \\
\hline polystyrene & $1.68 \mathrm{E}+09$ & 0.33 \\
\hline
\end{tabular}

Table 1. Bulk properties of substrate materials.

\section{CONCLUSIONS AND FUTURE WORK}

This new MEMS/Materials characterization system is capable of simultaneously measuring small displacements and deformations, six orders of magnitude in the force regime between typical atomic force microscope (AFM) cantilevers and conventional methods, and six orders of magnitude in contact resistance. When combined with materials data like focused ion beam sections for microstructure, AFM scans for surface roughness, and nanoindentation measurements for hardness, this system enables the understanding of thin film electrical contact behavior in the low force regime. Further investigation of materials properties and variation of contact parameters is underway to provide design rules for low force, thin film gold contacts.

\section{ACKNOWLEDGMENTS}

This work was supported through Formfactor, Inc., the Hertz Foundation Fellowship, and the Alliance for Innovative Manufacturing at Stanford. Assistance and advice from Kenny group members and staff at Formfactor and Stanford is gratefully acknowledged. Travel support has been generously provided by the Transducers Research Foundation and by the DARPA BioFlips programs.

\section{REFERENCES}

1. D. Hyman and M. Mehregany, "Contact physics of gold microcontacts for MEMS switches", Proceedings of the FortyFourth IEEE Holm Conference on Electrical Contacts, IEEE: New York, NY, USA (1998), pp. 325.

2. E. J. J. Kruglick and K. S. J. Pister, "Lateral MEMS microcontact considerations", J. Microelectromech. Syst. (USA), 8(3) 264-271 (1999).

3. P. M. M. Zavracky, Nicol E.; Morrison, Richard H.; Potter, David, "Microswitches and microrelays with a view toward microwave applications", Int J RF Microwave Comput Aided Eng, 9(4) 338-347 (1999).

4. J. Schimkat, "Contact measurements providing basic design data for microrelay actuators", in Sens. Actuators A, Phys.

(Switzerland), Sensors and Actuators A (Physical). (1999). 5. S. Hannoe and H. Hosaka, "Electrical characteristics of micro mechanical contacts", Microsystem Technologies, 3(1) 31-5 (1996).

6. S. C. Bromley and B. J. Nelson, "Performance of microcontacts tested with a novel MEMS device", Electrical Contacts,

Proceedings of the Annual Holm Conference on Electrical Contacts, pp. 122-127 (2001).

7. J. Beale and R. F. Pease, "Limits of high-density, low-force pressure contacts", IEEE Transactions on Components,

Packaging, and Manufacturing Technology, Part A, 17(2) 257-62 (1994).

8. M. Antler, "Tribological Properties of Gold For Electric Contacts", IEEE Transactions on Parts, Hybrids and Packaging, (1973).

9. R. Holm and E. Holm, "Electric contacts; theory and application". 4th completely rewritten ed., Berlin, New York, Springer-Verlag. xv, 482 (1967)

10. W. C. Oliver, R. Hutchings, and J. B. Pethica, "Measurement of Hardness At Indentation Depths As Low As 20 Nanometres", ASTM Special Technical Publication, (1985).

11. M. D. Pashley and J. B. Pethica, "The role of surface forces in metal-metal contacts", in J. Vac. Sci. Technol. A, Vac. Surf. Films (USA). (1985).

12. S. P. Sharma, "Some notes on the physics of contact adhesion", Insulation/Circuits, 23(11) 41-5 (1977).

13. B. Pruitt, A. Partridge, M. Bartsch, et al. "Design of piezoresistive cantilevers for low force electrical contact measurements", IMECE: 2000 International Mechanical Engineering Congress and Exposition, Orlando, FL, (2000). 14. E. Hecht and A. Zajac, "Optics", Reading: Addison-Wesley (1974).

15. B. Pruitt, D.-H. Choi, J. Florando, et al. "Low force electrical contact measurements using piezoresistive cantilevers to characterize thin-film metallization", Transducers '01/EuroSensors $X V$, Munich, Germany, Springer (2001), pp. 1032-35. 